\title{
Performance of GaN-Based LEDs with Nanopatterned Indium Tin Oxide Electrode
}

\author{
Zhanxu Chen, ${ }^{1}$ Wenjie Liu, ${ }^{2}$ Wei Wan, ${ }^{1}$ Gengyan Chen, ${ }^{3}$ Yongzhu Chen, ${ }^{3}$ \\ Baijun Zhang, ${ }^{4}$ and Chongjun Jin ${ }^{2}$ \\ ${ }^{1}$ School of Electronic and Information, Guangdong Polytechnic Normal University, Guangzhou 510665, China \\ ${ }^{2}$ State Key Laboratory of Optoelectronic Materials and Technologies, School of Materials Science and Engineering, \\ Sun Yat-Sen University, Guangzhou 510275, China \\ ${ }^{3}$ College of Electromechanical Engineering, Guangdong Polytechnic Normal University, Guangzhou 510635, China \\ ${ }^{4}$ School of Electronics and Information Technology, Sun Yat-Sen University, Guangzhou 510275, China
}

Correspondence should be addressed to Wenjie Liu; liuwenjie@mail.sysu.edu.cn and Baijun Zhang; zhbaij@mail.sysu.edu.cn

Received 27 June 2016; Accepted 16 August 2016

Academic Editor: Jia Liu

Copyright (C) 2016 Zhanxu Chen et al. This is an open access article distributed under the Creative Commons Attribution License, which permits unrestricted use, distribution, and reproduction in any medium, provided the original work is properly cited.

\begin{abstract}
The indium tin oxide (ITO) has been widely applied in light emitting diodes (LEDs) as the transparent current spreading layer. In this work, the performance of GaN-based blue light LEDs with nanopatterned ITO electrode is investigated. Periodic nanopillar ITO arrays are fabricated by inductive coupled plasma etching with the mask of polystyrene nanosphere. The light extraction efficiency (LEE) of LEDs can be improved by nanopatterned ITO ohmic contacts. The light output intensity of the fabricated LEDs with nanopatterned ITO electrode is $17 \%$ higher than that of the conventional LEDs at an injection current of $100 \mathrm{~mA}$. Three-dimensional finite difference time domain simulation matches well with the experimental result. This method may serve as a practical approach to improving the LEE of the LEDs.
\end{abstract}

\section{Introduction}

Recently, GaN-based light emitting diodes (LEDs) have been garnering an increasing amount of attention in the field of solid state lighting, signaling, and displays due to their broad emission wavelength range [1]. However, when considering LEDs lighting as a replacement for fluorescent lighting, their light extraction efficiency (LEE) is still relatively low due to the internal total reflection of light trapped inside LEDs [2]. To increase the LEE of LEDs, several approaches had focused on fabricating micro-/nanostructures either inside or outside the LEDs [3-20]. For example, roughed GaN surface [3-7], roughed sapphire substrate $[8,9]$, roughed indium tin oxide (ITO) surface [10-12], and grown $\mathrm{ZnO}$ micro-/nanostructures [13-15] have been implemented in LEDs. The common nanoscale patterning techniques, such as electron-beam lithography [16], nanoimprint lithography [17], holographic lithography [18], hydrothermally method
[19], and nanosphere lithography (NSL) [20], are used to obtain nanoscale surface textures.

Compared to the other techniques, NSL has the advantages of low cost and high throughput, which is very suitable for surface patterning. In the previous works, the twodimensional photonic crystal structure was fabricated on an InGaN/GaN multiple quantum well structure by a silica nanosphere lithography, and several times enhancement in photoluminescence intensity was observed [21, 22]. However, there was no research on the electroluminescence. Comparing to the silica nanosphere, the size of polystyrene (PS) nanosphere will be decreased with increasing the etching time as an etching mask. Through the PS NSL method, nanopillars with different diameters can be obtained and the LEE of LEDs can be optimized [23]. In addition, the nanopillar structure will take the characteristic of small top and big bottom, and this is helpful for the light to escape due to the gradient refractive index. In order to reduce 


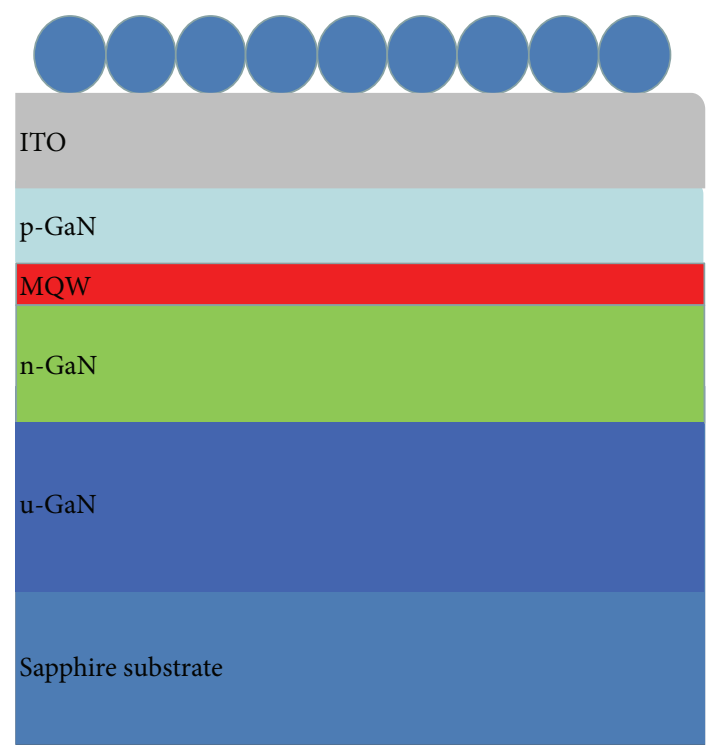

(a)

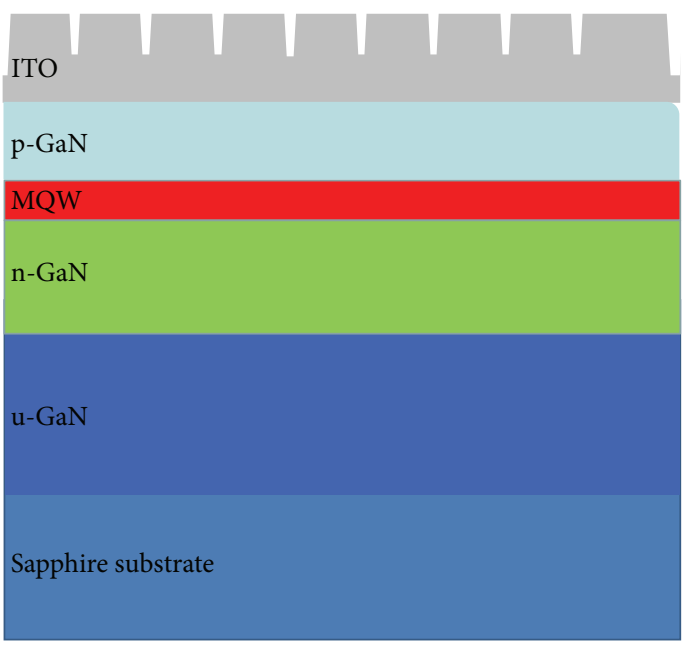

(c)

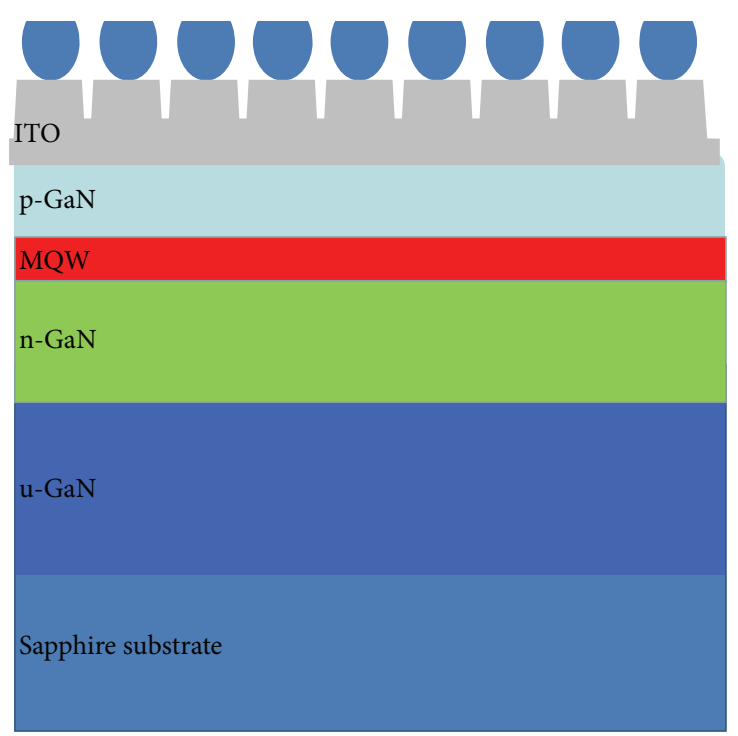

(b)

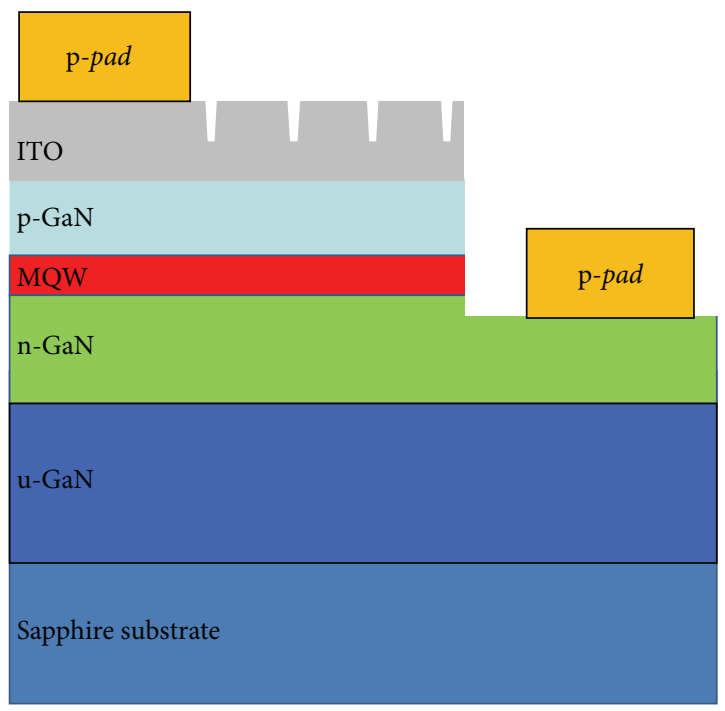

(d)

FIGURE 1: Schematic fabrication process for nanopatterned ITO electrode. (a) Deposition of PS spheres on the ITO electrode surface. (b) ICP etching to nanopatterned ITO electrode. (c) Removal of the PS nanospheres. (d) Schematic illustration of the GaN-based blue LEDs with nanopatterned ITO electrode.

the influence of etching on the electrical characteristics of the LED device, the nanostructures should be prepared on the ITO transparent electrode layer. However, this research has rarely been reported. In this work, by PS NSL technology, GaN-based blue LEDs with surface patterned ITO electrode were fabricated and the optical and electrical performances of the LEDs with nanopatterned ITO electrode were analyzed and discussed. The electroluminescence intensity of the ITO patterned LEDs is increased by $17 \%$ at $100 \mathrm{~mA}$ injection current compared to that of conventional LEDs. Finally, the light output enhancements are simulated based on three-dimensional finite difference time domain (3D-FDTD) method to verify the experimental results.

\section{Experimental Methods}

The GaN-based $(\lambda=465 \mathrm{~nm})$ epitaxial wafer was grown on a 2-inch sapphire $\left(\mathrm{Al}_{2} \mathrm{O}_{3}\right)$ substrate using a metal-organic chemical vapor deposition. After the growth of a $2 \mu \mathrm{m}$ undoped $\mathrm{GaN}$ (u-GaN) buffer layer and $3 \mu \mathrm{m} \mathrm{n}-\mathrm{GaN}$ layer, an active layer of five-period InGaN/GaN MQWs and a $150 \mathrm{~nm}$ p-GaN layer were deposited. The device fabrication process was as follows. A transparent ITO electrode with a thickness of about $400 \mathrm{~nm}$ was first deposited on the p-GaN surface. Then, the LED chips with dimensions of $300 \mu \mathrm{m} \times 300 \mu \mathrm{m}$ were formed by mesa-etching the exposed n-type GaN via standard lithography, ITO wet etching, 


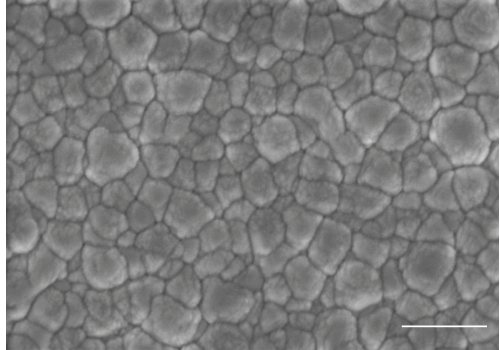

(a)

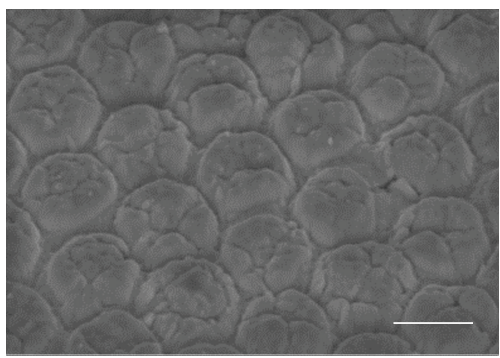

(d)

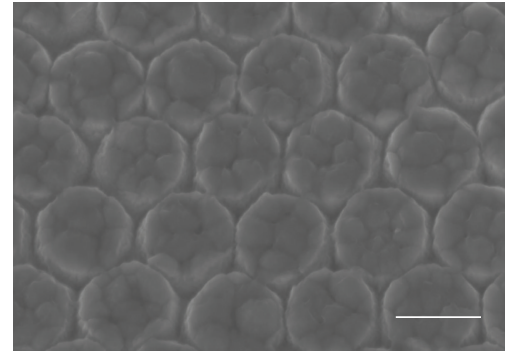

(b)

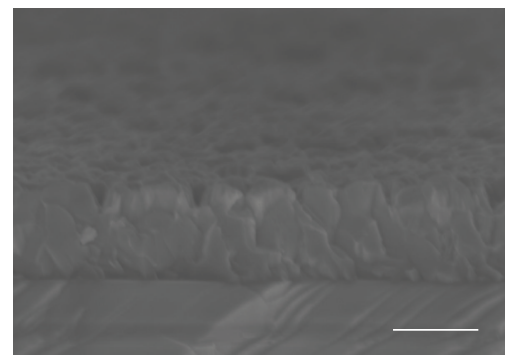

(e)

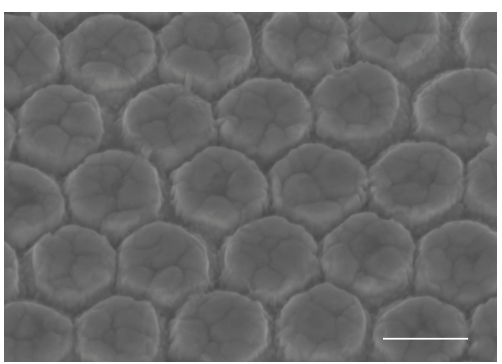

(c)

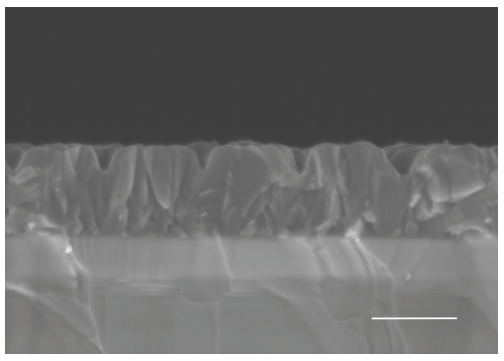

(f)

FIGURE 2: SEM images of the nanopillar patterned ITO layer. Scale bars are $400 \mathrm{~nm}$. (a) The original surface of ITO layer. (b)-(d) $30^{\circ}$ tilt SEM images of samples A, B, and C, respectively. (e)-(f) Cross-sectional image of samples B and C. The ITO layers of samples A, B, and C were etched by ICP etching for $60 \mathrm{~s}, 80 \mathrm{~s}$, and $100 \mathrm{~s}$, respectively.

and subsequent inductively coupled plasma (ICP) etching. $\mathrm{Cr} / \mathrm{Pt} / \mathrm{Au}(200 / 400 / 2000 \mathrm{~nm})$ was finally deposited on the top of ITO surface as well as the exposed n-GaN layer as a contact metal for both the p- and the n-GaN layers.

After the formation of the contact metal electrodes on the $\mathrm{p}$ - and the $\mathrm{n}-\mathrm{GaN}$ layers, the nanopatterned ITO layer was fabricated through the NSL, and the process flow was shown in Figure 1. First, a hexagonal close-packed monolayer of PS nanospheres with a $450 \mathrm{~nm}$ diameter was formed on the ITO layer, as shown in Figure 1(a). Second, the ITO layer with a monolayer mask of PS spheres was etched by an ICP etching machine via the gas flow of $\mathrm{BCl}_{3} / \mathrm{Cl}_{2} / \mathrm{Ar}$, as shown in Figure 1(b). Finally, the PS spheres were removed by trichloromethane solvent with sonication and the periodic ITO nanopillar arrays were obtained, as shown in Figure 1(c). Figure 1(d) presented the schematic of the modified LED structure with a nanopillar patterned ITO electrode.

\section{Results and Discussion}

By the method described above, we could fabricate the LEDs with periodic ITO nanopillar arrays. When the ITO was etched by ICP, the mask of PS spheres was also etched and the size of the PS spheres was altered. Therefore, various height and diameter of the top part of the ITO nanopillar could be obtained by changing the ICP etching time. In order to explore the different effects of various ITO nanopillar structure on LEE of the LED, three nanopatterned ITO samples were fabricated by different ICP etching time. The three samples with the nanopillar ITO layer etched by ICP for $60 \mathrm{~s}, 80 \mathrm{~s}$, and $100 \mathrm{~s}$ were marked as samples $\mathrm{A}, \mathrm{B}$, and $\mathrm{C}$, respectively. The three nanopatterned ITO samples and conventional LEDs were fabricated from the same InGaN/GaN LED wafer to eliminate the differences in the device characteristics. Figure 2 shows scanning electron microscope (SEM) images of ITO layer of samples A, B, and $\mathrm{C}$ and the conventional LED. As shown in Figures 2(b)2(d), when the ICP etching time is longer, the surface of ITO is etched more obviously and the height of the nanopillars becomes higher. The diameters of nanopillars for samples A, $\mathrm{B}$, and $\mathrm{C}$ are found to be about $400 \mathrm{~nm}, 390 \mathrm{~nm}$, and $380 \mathrm{~nm}$ from the SEM images, respectively. Figures 2(e) and 2(f) show cross-sectional images of samples $\mathrm{B}$ and $\mathrm{C}$. The heights of the nanopillars of samples A, B, and C are about $60 \mathrm{~nm}, 100 \mathrm{~nm}$, and $140 \mathrm{~nm}$, respectively.

Figure 3(a) shows the light output intensity (LOI) as functions of injection current for the three nanopatterned LEDs and a conventional LED. At the same injection current, the light output intensity of the three nanopatterned LEDs is higher than that of the conventional LED. At an operating current of $100 \mathrm{~mA}$, the light output intensity of the samples $\mathrm{A}, \mathrm{B}$, and $\mathrm{C}$ is approximately $3 \%, 14 \%$, and $19 \%$ higher than that of the conventional LED, respectively. Figure 3(b) shows the forward current-voltage $(I-V)$ characteristics of the four samples. It is clear that the LED with nanopatterned LEDs exhibits nearly the same $I-V$ characteristics as the conventional LED. The forward voltages at $100 \mathrm{~mA}$ are 4.42 , $4.46,4.46 \mathrm{~V}$, and 4.37 for the samples $\mathrm{A}, \mathrm{B}$, and $\mathrm{C}$ and conventional LED, which consequently indicates an acceptable electrical performance for the nanopatterned LEDs. In order to eliminate differences in input electric power (IEP) of the samples, the IEP-LOI curves were calculated from the curves in Figures 3(a) and 3(b). As shown in Figure 3(c), the LOI of the three nanopatterned LEDs are higher than that of the 


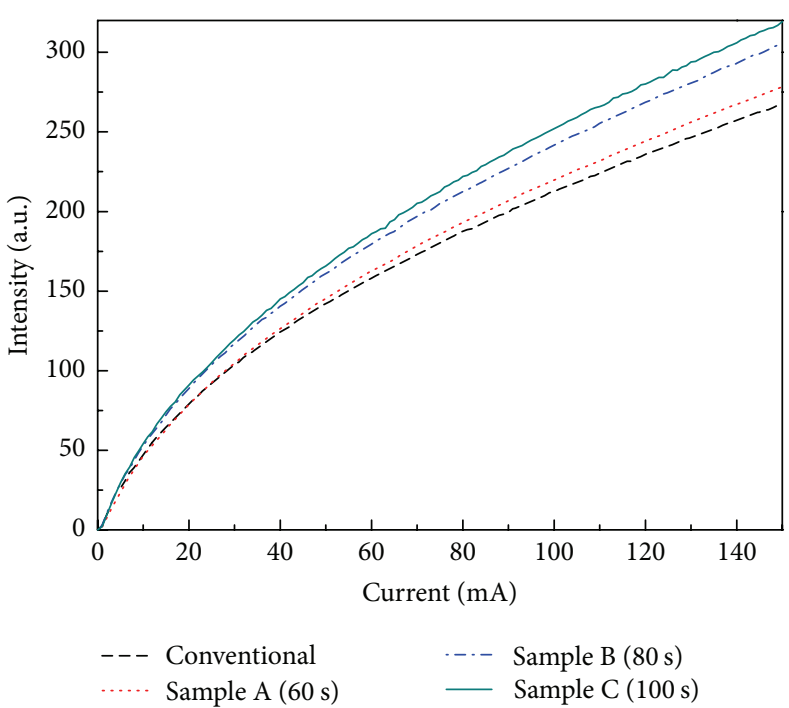

(a)

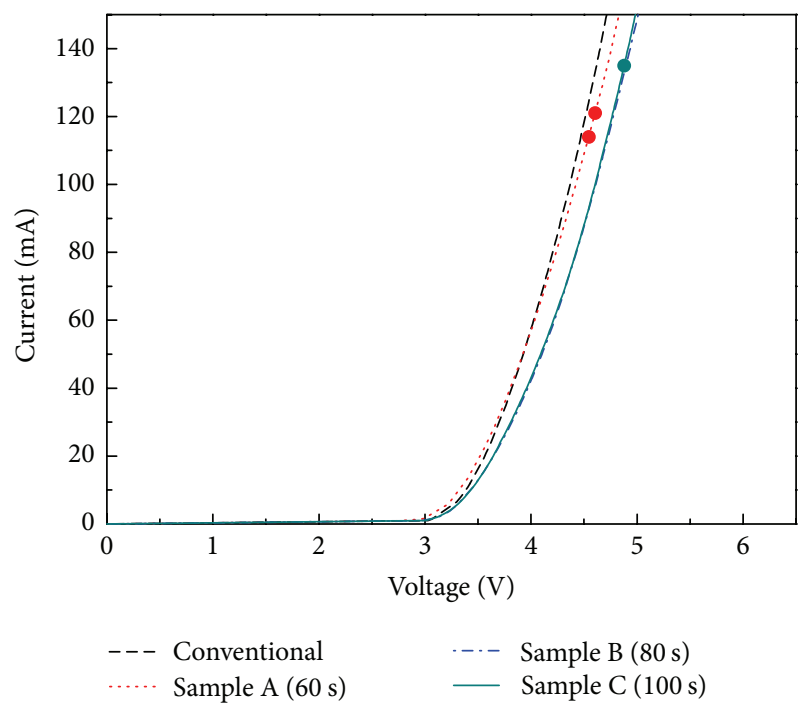

(b)

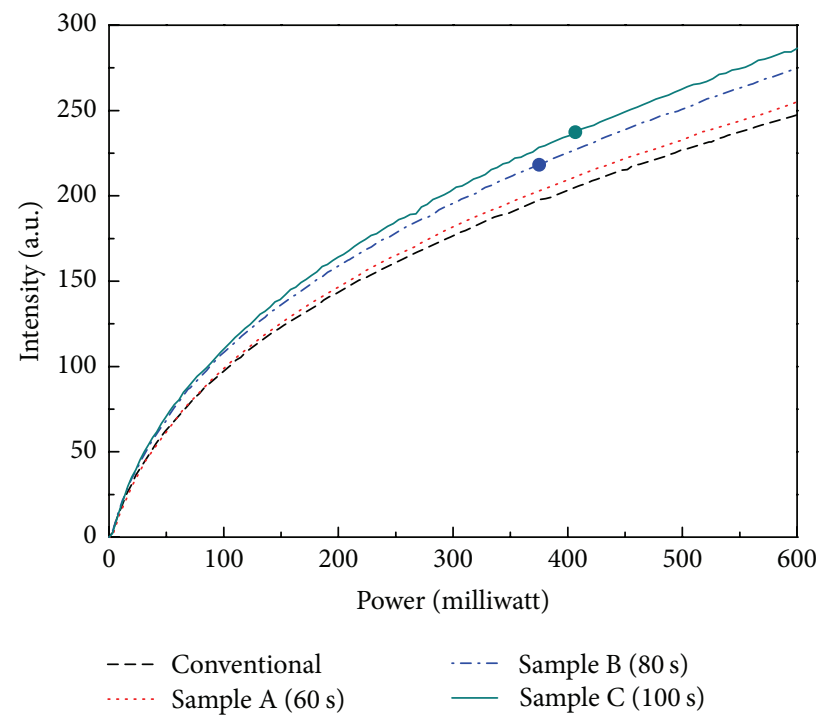

(c)

FIGURE 3: Electroluminescence curves of three nanopillar patterned LEDs and a conventional LED. (a) The optical output power versus injection current $(O-I)$ characteristics. (b) The current versus voltage $(I-V)$. (c) The optical output power versus power $(O-P)$ characteristics.

conventional LED at the whole IEP range. At an operating current of $100 \mathrm{~mA}$, the LOI of the samples A, B, and C are approximately $3 \%, 12 \%$, and $17 \%$ higher than that of the conventional LED, respectively. Therefore, we believe that the proposed technology is an effective method to improve the LEE of GaN-based LEDs.

The results showed that the LEE of the samples with nanopillar patterned structures were enhanced. With increasing the etching depth, the size of the nanopillar became smaller, and the light extraction efficiency of the sample would be relatively higher. With the nanopillar patterned ITO surface, photons should experience multiple scattering at the sample surface and could escape from the device easily, as shown in Figure 4. For the LEDs with the flat surface ITO structure, the emitted light having the incident angle smaller than the critical angle was the only light that can be extracted, as shown in Figure 4(a). According to Snell's law, the critical angle $\theta_{c}$ of the total internal reflection satisfied the formula $\sin \theta_{c}=n_{2} / n_{1}$, where $n_{1}=1.9$ and $n_{2}=1$ are the refractive indexes of ITO and air, respectively. Then, the critical angles of total reflection at air/ITO interface are around $31.8^{\circ}$. Thus, the majority of photons are reflected from the interface of conventional ITO LEDs [17]. The nanopillar patterned LEDs have higher LEE, which can be explained from various points of view. Firstly, the nanopillar array played the role of roughness. Secondly, the periodic nanopillar array served as a two-dimensional grating. Such Bragg scattering assisted the waveguide modes to become the radiation mode; then 


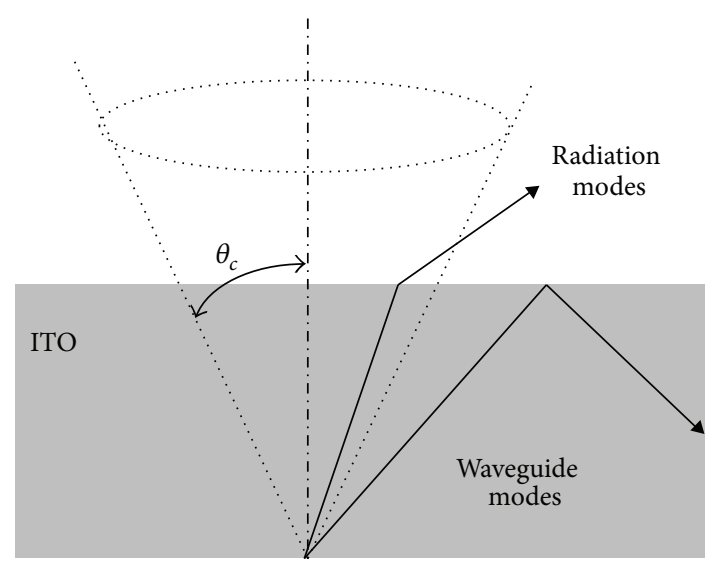

(a)

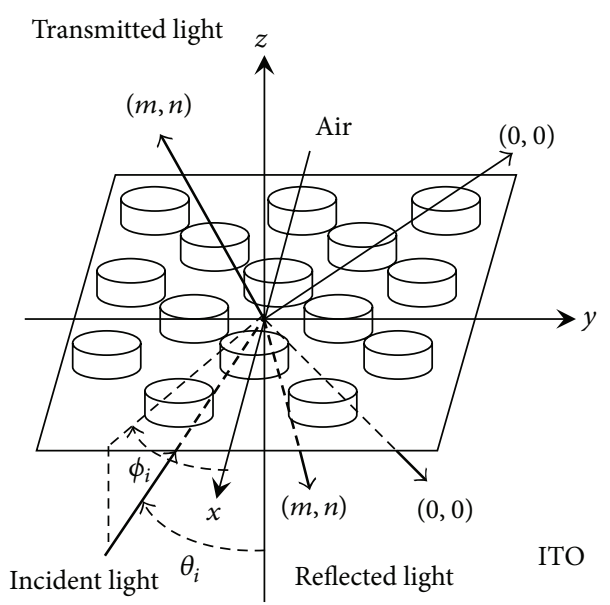

(b)

FIGURE 4: Light emission of (a) conventional ITO LEDs and (b) LEDs with nanopatterned ITO electrode.

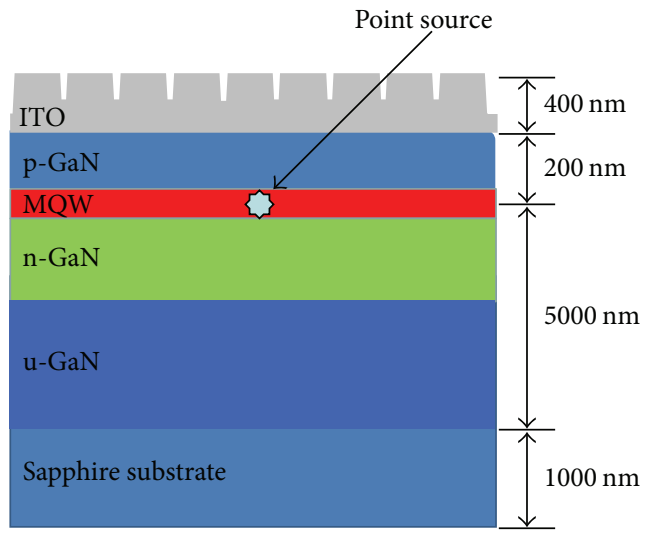

(a)

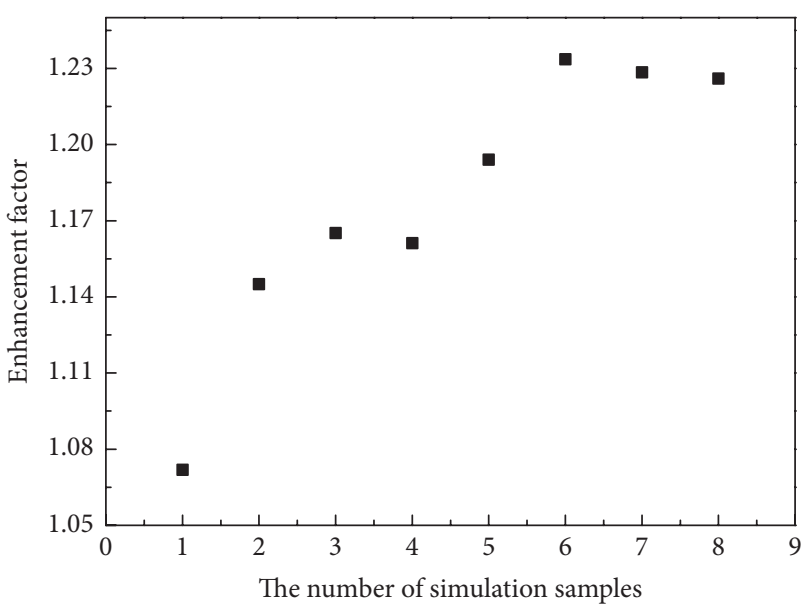

(b)

FIGURE 5: (a) Schematic illustration of LED simulation model. (b) The 3D-FDTD simulation result, in which the $X$-axis is the serial number of the simulation samples, and the $Y$-axis is the increase ratio of LEE compared with reference sample.

the photons have taken more opportunities to escape from the ITO surface into the air, as shown in Figure 4(b). The transmitted and reflected light can be expressed as [24]

$$
\begin{aligned}
& \frac{n_{2}}{\lambda} \sin \theta_{t}\left(\cos \phi_{t}, \sin \phi_{t}\right) \\
& \quad=\frac{n_{1}}{\lambda}\left(\sin \theta_{i}\right)\left(\cos \phi_{i}, \sin \phi_{i}\right)+\left(\frac{m}{\Lambda_{x}}, \frac{n}{\Lambda_{y}}\right), \\
& \frac{n_{1}}{\lambda} \sin \theta_{r}\left(\cos \phi_{r}, \sin \phi_{r}\right) \\
& \quad=\frac{n_{1}}{\lambda}\left(\sin \theta_{i}\right)\left(\cos \phi_{i}, \sin \phi_{i}\right)+\left(\frac{m}{\Lambda_{x}}, \frac{n}{\Lambda_{y}}\right) .
\end{aligned}
$$

$n_{1}$ and $n_{2}$ are refractive indices of ITO and air, respectively. $\lambda$ is the wavelength of light in the vacuum. $\Lambda_{x}$ and $\Lambda_{y}$ are periods of the lattice in the $x$ and $y$ directions. $m$ and $n$ are integers indicating the diffraction orders. $\theta_{i}, \theta_{t}$, and $\theta_{r}$ are the incident angle, transmitted angle, and reflected angle, respectively. $\phi_{i}, \phi_{t}$, and $\phi_{r}$ are the azimuthal angle of incident light, transmitted light, and reflected light, respectively. So, the waveguide modes can be coupled to the radiation modes. This will make the photons that were originally emitted out of an escaping cone go back into the escaping cone and improve the LEE of the LEDs.

In order to verify the experiments, we also perform 3D-FDTD simulations to give the light extraction of the LEDs with nanostructures. The simulated LED structure is shown in Figure 5(a), which consisted of a $1000 \mathrm{~nm}$ sapphire substrate, a $5200 \mathrm{~nm} \mathrm{GaN}$ layer (including the $\mathrm{u}-\mathrm{GaN}, \mathrm{MQW}$, and $\mathrm{p}-\mathrm{GaN}$ layer), and a $400 \mathrm{~nm}$ ITO layer. In simulation, the lateral space of simulation region is $8000 \mathrm{~nm} \times 8000 \mathrm{~nm}$, and there are about $18 \times 20$ nanopillars in the simulation region. To explore the effect of the diameters and heights of the nanopillars on the LEE, eight samples are simulated 
here. For samples 1, 2, and 3, the diameters are set as $400 \mathrm{~nm}$, $390 \mathrm{~nm}, 380 \mathrm{~nm}$, and the heights are set as $60 \mathrm{~nm}, 100 \mathrm{~nm}$, and $140 \mathrm{~nm}$, respectively. For samples 4-8, the diameters of the nanopillars are set as $380 \mathrm{~nm}$ and the heights are set as $180 \mathrm{~nm}, 220 \mathrm{~nm}, 260 \mathrm{~nm}$, and $300 \mathrm{~nm}$, respectively. The light extraction effect of LED with different nanostructures is simulated by the $3 \mathrm{D}$-FDTD method. In the simulation, the wavelength of incident light is $465 \mathrm{~nm}$, which corresponds to the center wavelength of emission spectrum. The refractive indices of GaN and ITO are approximately 2.49 and 1.9 at the wavelength of $465 \mathrm{~nm}$. The simulation results are shown in Figure 5(b). The horizontal coordinate is the number of simulated samples, and the longitudinal coordinate is the increase ratio of LEE. The simulation results show that the LEE of samples 1-3 is increased, which is consistent with the experimental results. In addition, we also see that simulated sample 3 (height of $140 \mathrm{~nm}$ ) is a local optimal value, and the structure of the optimal value is near sample 6 (height of $260 \mathrm{~nm})$. This is consistent with the rough calculation [25]

$$
h=\frac{\lambda}{n_{\mathrm{ITO}-\mathrm{pc}}} \approx \frac{465}{1.64} \approx 284 \mathrm{~nm},
$$

where $\lambda$ is the incident light wavelength and $n_{\text {ITO-pc }}$ is the effective refractive index of the ITO nanostructures layer.

\section{Conclusions}

Benefiting from the excellent electrical conductivity and light transmittance, ITO transparent electrode has replaced nickel gold alloy as a transparent electrode of the LEDs. However, the refractive index of ITO is 1.9 and is much higher than the refractive index of air, which limits the light escaping from LEDs. In this paper, we fabricated LEDs with nanopillar patterned ITO layer via nanosphere lithography. The optical and electrical performances of the LEDs with nanopatterned ITO electrode were investigated. The results show that the LEE was enhanced with increasing the etching depth. The electroluminescence intensity of the ITO patterned LEDs was increased by $17 \%$ at $100 \mathrm{~mA}$ injection current compared to that of conventional LEDs. The enhancement of the LEE can be ascribed to the fact that the total reflection of the ITO surface is broken by the periodic nanopillars structure. The LEE may be further improved by optimizing the nanopillars structure. Therefore, this is a promising method for realizing high-efficiency LEDs.

\section{Competing Interests}

The authors declare that there are no competing interests regarding the publication of this paper.

\section{Acknowledgments}

This work was partly supported by the National Natural Science Foundation of China (11374376, 11174374, 11574406, 11504058, and 61475038) and the Guangdong Province Natural Science Foundation of China (2015A030310213).

\section{References}

[1] E. F. Schubert and J. K. Kim, "Solid-state light sources getting smart," Science, vol. 308, no. 5726, pp. 1274-1278, 2005.

[2] C.-F. Lin, J.-H. Zheng, Z.-J. Yang et al., "High-efficiency InGaNbased light-emitting diodes with nanoporous GaN:Mg structure," Applied Physics Letters, vol. 88, no. 8, Article ID 083121, 2006.

[3] G.-D. Hao, A. M. Jahir, T. Takahashi et al., "Enhanced light extraction in GaN-based light-emitting diodes by evanescent wave coupling effect," Applied Physics Express, vol. 7, no. 10, Article ID 102101, 2014.

[4] J. J. Wierer Jr., A. David, and M. M. Megens, "III-nitride photonic-crystal light-emitting diodes with high extraction efficiency," Nature Photonics, vol. 3, no. 3, pp. 163-169, 2009.

[5] H.-M. An, J. I. Sim, K. S. Shin, Y. M. Sung, and T. G. Kim, "Increased light extraction from vertical GaN light-emitting diodes with ordered, cone-shaped deep-pillar nanostructures," IEEE Journal of Quantum Electronics, vol. 48, no. 7, pp. 891-896, 2012.

[6] J.-H. Park, J.-W. Park, I.-K. Park, and D.-Y. Kim, "Enhanced optical power of GaN-based light-emitting diode with nanopatterned p-GaN by simple light coupling mask lithography," Applied Physics Express, vol. 5, no. 2, Article ID 022101, 2012.

[7] Y. Zhang, T. Wei, Z. Xiong et al., "Enhanced optical power of GaN-based light-emitting diode with compound photonic crystals by multiple-exposure nanosphere-lens lithography," Applied Physics Letters, vol. 105, no. 1, Article ID 013108, 2014.

[8] C.-C. Kao, Y.-K. Su, C.-L. Lin, and J.-J. Chen, "The aspect ratio effects on the performances of GaN-based light-emitting diodes with nanopatterned sapphire substrates," Applied Physics Letters, vol. 97, no. 2, Article ID 023111, 2010.

[9] J.-Y. Cho, J.-S. Kim, Y.-D. Kim, H. J. Cha, and H. Lee, "Fabrication of oxide-based nano-patterned sapphire substrate to improve the efficiency of GaN-based of LED," Japanese Journal of Applied Physics, vol. 54, no. 2, Article ID 02BA04, 2015.

[10] S. M. Huang, Y. Yao, C. Jin, Z. Sun, and Z. J. Dong, "Enhancement of the light output of GaN-based light-emitting diodes using surface-textured indium-tin-oxide transparent ohmic contacts," Displays, vol. 29, no. 3, pp. 254-259, 2008.

[11] Y. Zhang, T. Wei, Z. Xiong et al., "Enhancing optical power of GaN-based light-emitting diodes by nanopatterning on indium tin oxide with tunable fill factor using multiple-exposure nanosphere-lens lithography," Journal of Applied Physics, vol. 116, no. 19, Article ID 194301, 2014.

[12] S. H. Jung, K. M. Song, Y. S. Choi et al., "Light output enhancement of InGaN/GaN light-emitting diodes with contrasting indium tin-oxide nanopatterned structures," Journal of Nanomaterials, vol. 2013, Article ID 832170, 6 pages, 2013.

[13] K. S. Kim, S.-M. Kim, H. Jeong, M. S. Jeong, and G. Y. Jung, "Enhancement of light extraction through the wave-guiding effect of $\mathrm{ZnO}$ sub-microrods in InGaN blue light-emitting diodes," Advanced Functional Materials, vol. 20, no. 7, pp. 10761082, 2010.

[14] Z. Yin, X. Liu, H. Yao et al., "Light extraction enhancement of GaN LEDs by hybrid ZnO micro-cylinders and nanorods array," IEEE Photonics Technology Letters, vol. 25, no. 20, pp. 1989-1992, 2013.

[15] B. D. Ryu, P. Uthirakumar, J. H. Kang et al., "Enhanced light output power of GaN-based light-emitting diodes by nanorough indium tin oxide film using $\mathrm{ZnO}$ nanoparticles," Journal of Applied Physics, vol. 109, no. 9, Article ID 093116, 2011. 
[16] J. J. Wierer, M. R. Krames, J. E. Epler et al., "InGaN/GaN quantum-well heterostructure light-emitting diodes employing photonic crystal structures," Applied Physics Letters, vol. 84, no. 19, pp. 3885-3887, 2004.

[17] S. J. Chang, C. F. Shen, W. S. Chen et al., "Nitride-based light emitting diodes with indium tin oxide electrode patterned by imprint lithography," Applied Physics Letters, vol. 91, no. 1, Article ID 013504, 2007.

[18] S.-M. Pan, R.-C. Tu, Y.-M. Fan, R.-C. Yeh, and J.-T. Hsu, "Improvement of InGaN-GaN light-emitting diodes with surface-textured indium-tin-oxide transparent ohmic contacts," IEEE Photonics Technology Letters, vol. 15, no. 5, pp. 649651, 2003.

[19] H. Jeong, D. J. Park, H. S. Lee et al., "Light-extraction enhancement of a GaN-based LED covered with $\mathrm{ZnO}$ nanorod arrays," Nanoscale, vol. 6, no. 8, pp. 4371-4378, 2014.

[20] T. S. Kim, S.-M. Kim, Y. H. Jang, and G. Y. Jung, "Increase of light extraction from GaN based light emitting diodes incorporating patterned structure by colloidal lithography," Applied Physics Letters, vol. 91, no. 17, Article ID 171114, 2007.

[21] M. Athanasiou, T. K. Kim, B. Liu, R. Smith, and T. Wang, "Fabrication of two-dimensional InGaN/GaN photonic crystal structure using a modified nanosphere lithography technique," Applied Physics Letters, vol. 102, no. 19, Article ID 191108, 2013.

[22] K. H. Li, Z. Ma, and H. W. Choi, “Tunable clover-shaped $\mathrm{GaN}$ photonic bandgap structures patterned by dual-step nanosphere lithography," Applied Physics Letters, vol. 100, no. 14, Article ID 141101, 2012.

[23] Z. X. Chen, Y. Ren, G. H. Xiao et al., "Enhancing light extraction of GaN-based blue light-emitting diodes by a tuned nanopillar array," Chinese Physics B, vol. 23, no. 1, Article ID 018502, 2014.

[24] H. Kikuta, S. Hino, A. Maruyama, and A. Mizutani, "Estimation method for the light extraction efficiency of light-emitting elements with a rigorous grating diffraction theory," Journal of the Optical Society of America A: Optics, Image Science, and Vision, vol. 23, no. 5, pp. 1207-1213, 2006.

[25] H. Gao, F.-M. Kong, K. Li, X.-L. Chen, Q.-A. Ding, and J. Sun, "Structural optimization of GaN blue light LED with double layers of photonic crystals," Acta Physica Sinica, vol. 61, no. 12, Article ID 127807, 2012. 

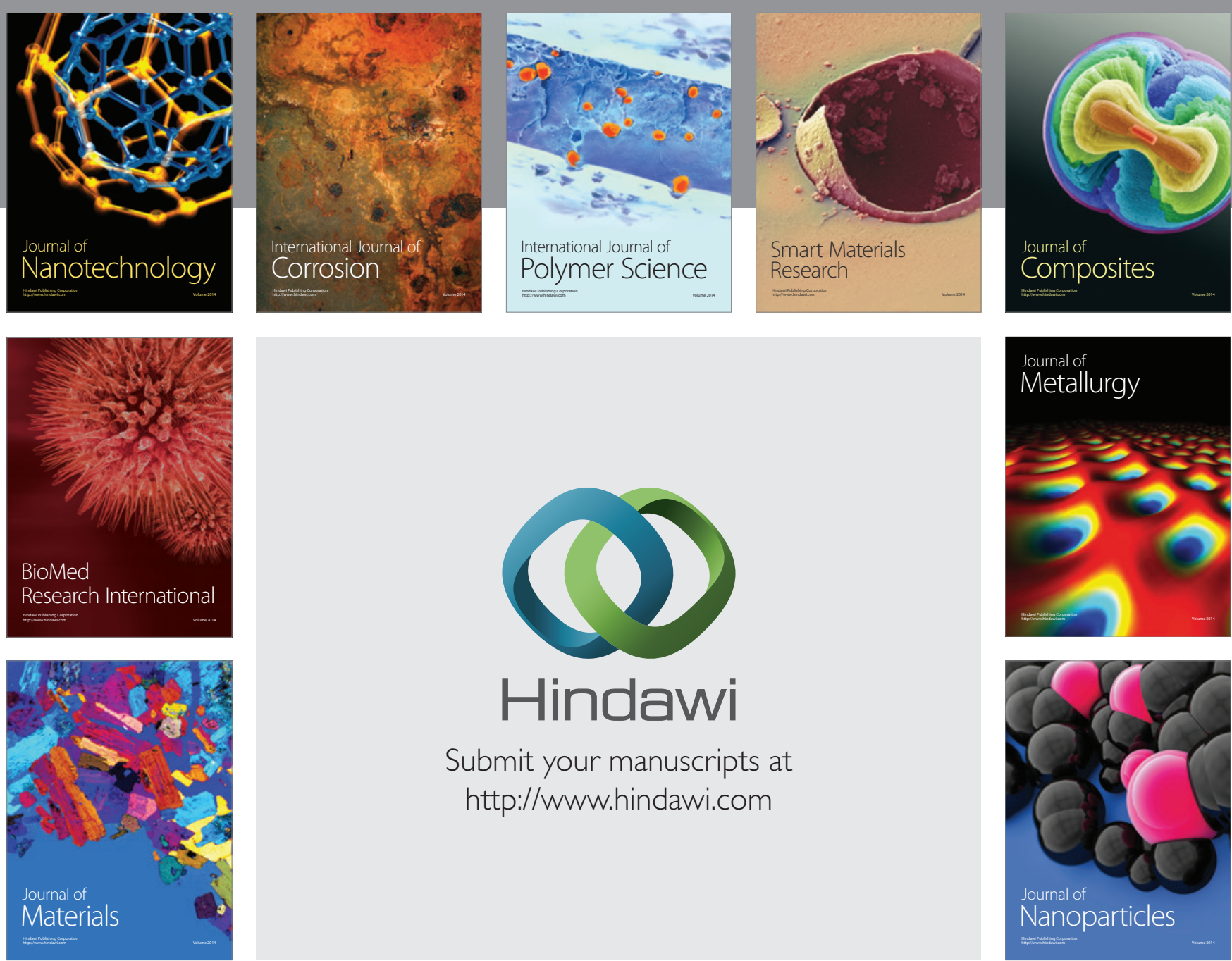

\section{Hindawi}

Submit your manuscripts at

http://www.hindawi.com

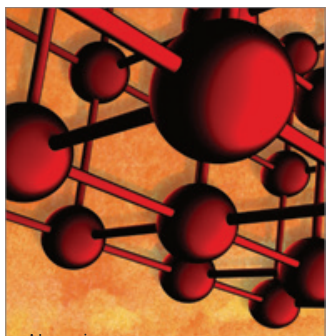

Materials Science and Engineering
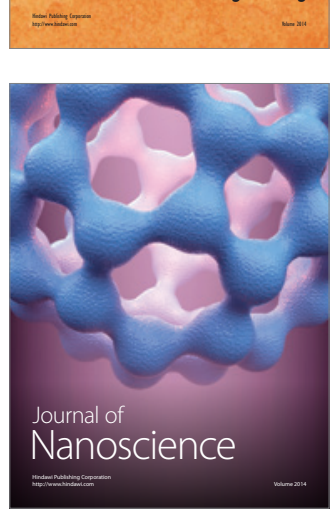
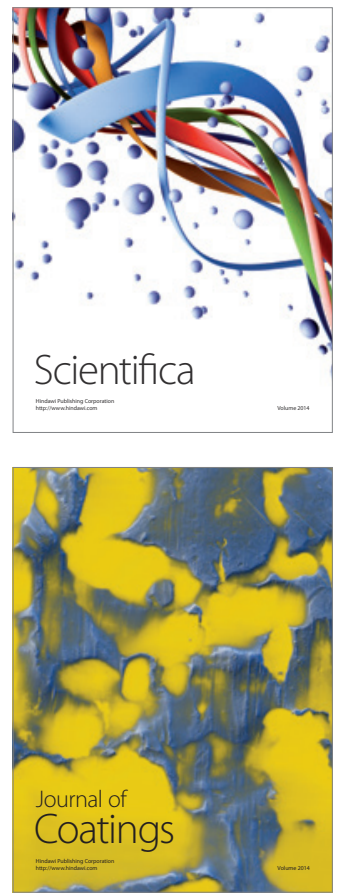
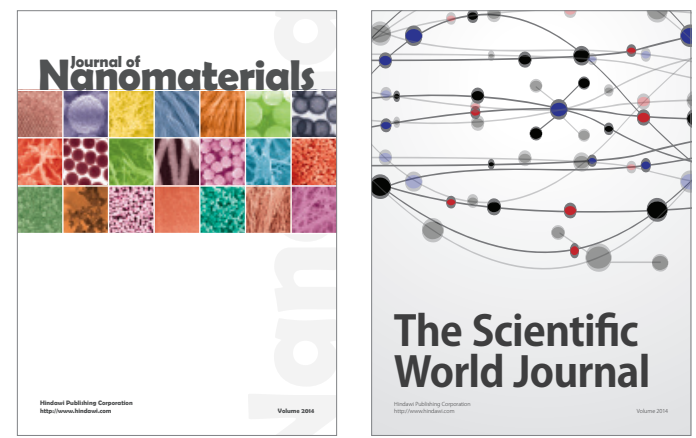

The Scientific World Journal
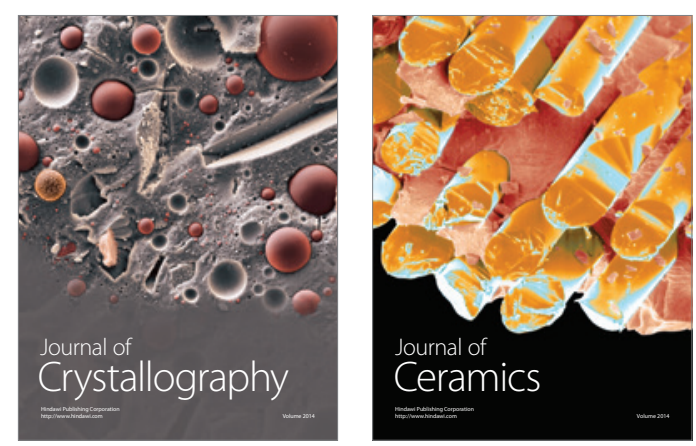
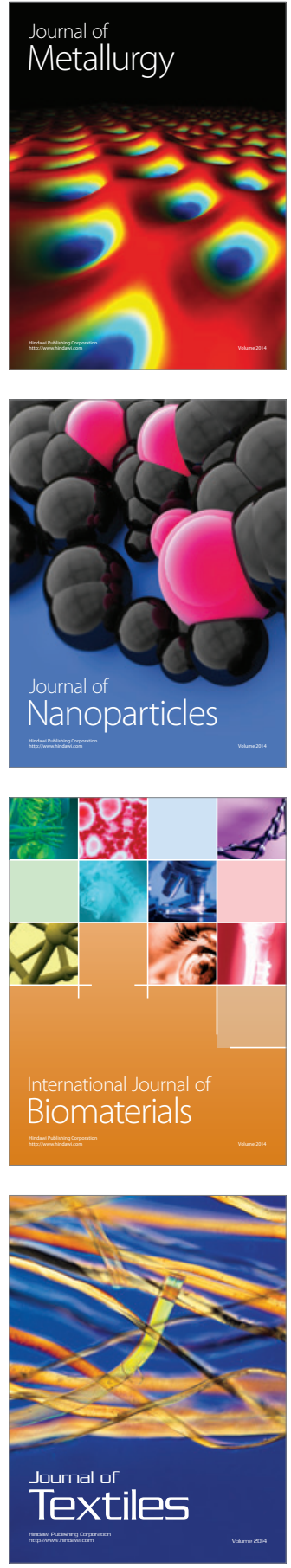